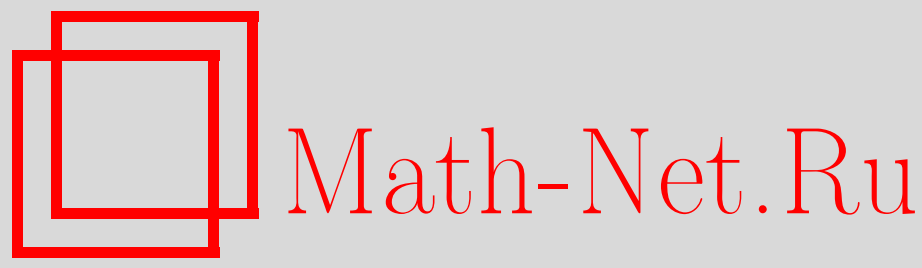

В. А. Березин, Классический аналог квантовой черной дыры Шварцшильда. "Стандартная модель" и за ее пределами, ТМФ, 2012, том 170, номер 1, 87-101

DOI: https://doi.org/10.4213/tmf6747

Использование Общероссийского математического портала Math-Net.Ru подразумевает, что вы прочитали и согласны с пользовательским соглашением http://www . mathnet.ru/rus/agreement

Параметры загрузки:

IP : 3.82 .47 .9

26 апреля 2023 г., $16: 16: 40$

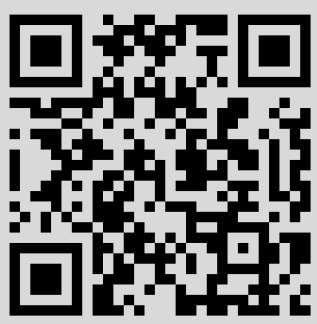




\title{
КЛАССИЧЕСКИЙ АНАЛОГ КВАНТОВОЙ ЧЕРНОЙ ДЫРЫ ШВАРЦШИЛЬДА. “СТАНДАРТНАЯ МОДЕЛЬ" И ЗА ЕЕ ПРЕДЕЛАМИ
}

\begin{abstract}
Построена модель, в которой основные глобальные свойства классических и квазиклассических черных дыр становятся локальными - это горизонт событий, отсутствие "волос", температура и энтропия. Конструкция базируется на особенностях квантового коллапса, обнаруженных при изучении некоторых конкретных моделей квантовых черных дыр. Однако предлагаемая модель чисто классическая, что позволяет использовать самосогласованным образом уравнения Эйнштейна и классическую (локальную) термодинамику и, в частности, разрешить "загадку $\ln 3$ ”.
\end{abstract}

Ключевые слова: квантовые черные дыры, термодинамика, квазинормальные частоты.

\section{1. ВВЕДЕНИЕ}

Классическое определение черной дыры основано на существовании горизонта событий [1] - границы пространственно-временно́й области, из которой свет не может вырваться на бесконечность. Само понятие горизонта событий является глобальным и требует знания всей истории, как прошлой, так и будущей.

Классическая "черная дыра не имеет волос" [2] и описывается лишь небольшим числом параметров: значениями массы, заряда кулоновского типа и углового момента. Черная дыра Шварцшильда обладает только массой, черная дыра Рейсснера-Нордстрема - массой и зарядом, а черная дыра Керра - массой и угловым моментом. Наиболее общей является черная дыра Керра-Ньюмена - она имеет все три параметра. Это напоминает описание тела, находящегося в состоянии теплового равновесия. Процесс "облысения" также является глобальным, формально он длится бесконечно, подобно процессу установления теплового равновесия. Этот процесс идет посредством распада и излучения всех возможных возмущений и описывается уравнением типа уравнения Шредингера, впервые выведенным в работе [3]. Результаты многих численных исследований в течение долгого времени (два десятилетия) были подытожены в монографии [4]. Оказалось, что моды этих возмущений имеют

* Институт ядерных исследований РАН, Москва, Россия. E-mail: berezin@ms2.inr.ac.ru 
дискретный спектр с комплексными частотами $w$, которые получили название "квазинормальные частоты". Мнимые части эквидистантны; это указывает на то, что распадающиеся моды излучаются подобно умирающим тонам звенящего колокола, и чем выше обертон, тем короче его время жизни. При этом действительные части квазинормальных частот стремятся к конечному пределу, величина которого зависит от значений параметров и типа черной дыры. Для черной дыры Шварцшильда, которую мы будем исследовать,

$$
G m w_{n}=0.0437123-\frac{i}{4}\left(n+\frac{1}{2}\right)+O\left[(n+1)^{-1 / 2}\right], \quad n \rightarrow \infty,
$$

где $m$ - масса черной дыры, а $G$ - ньютоновская гравитационная постоянная. Все это говорит о том, что черные дыры обладают некоторой встроенной собственной частотой. Следовательно, они вовсе не "мертвые", а ведут некую "частную жизнь", закодированную в свойствах их горизонтов событий. Очевидно, это тоже глобальное свойство, поскольку оно не зависит от происходящего внутри черной дыры.

Исследования процессов вблизи горизонта событий показали, что они, как и в термодинамике, могут быть обратимыми и необратимыми [5], [6]. Поглощение точечной (классической) частицы неэкстремальной черной дырой (при наличии более одного параметра существует минимальное значение массы, при фиксированных остальных параметрах - критическое, или экстремальное, ниже которого горизонт событий отсутствует) оказывается обратимым, если эта частица впрыскивается на горизонте событий как раз в точке поворота ее траектории. В этом случае площадь горизонта остается неизменной, а изменения других параметров (массы, заряда и углового момента) могут быть возвращены к первоначальным значениям другим обратимым процессом. Во всех остальных случаях площадь горизонта $A$ возрастает. Другими словами, для классических черных дыр $d A \geqslant 0$.

Новая эра в физике черных дыр началась с работы Бекенштейна [7], где представлены серьезные физические аргументы в пользу того, что черной дыре Шварцшильда должна быть приписана некоторая энтропия, величина которой пропорциональна площади горизонта событий. Такая строгая прямая пропорциональность могла бы показаться игрой с символами с одним только параметром, но она была подтверждена Бардиным, Картером и Хокингом [8], которые доказали справедливость четырех законов термодинамики для черных дыр общего вида - Керра-Ньюмена. Более того, было показано, что роль температуры играет (с точностью до численного множителя) постоянное значение поверхностной гравитации $\varkappa$ на горизонте событий. Но только после открытия Хокингом испарения черных дыр [9] эта термодинамическая аналогия стала рассматриваться как реальное физическое явление. Хокинг исследовал квантовую теорию безмассового скалярного поля на фоне статического пространства-времени Шварцшильда и обнаружил, что специфические граничные условия - существование только падающих волн вблизи горизонта - приводят к тепловому поведению волновых функций и ненулевому потоку энергии на бесконечности будущего. Оказалось, что спектр этого излучения планковский с температурой

$$
T_{\mathrm{H}}=\frac{\varkappa_{\mathrm{H}}}{2 \pi},
$$

где $\varkappa$ есть значение поверхностной гравитации на горизонте событий. Отсюда следует, что энтропия черной дыры равна в точности одной четверти безразмерной 
площади горизонта:

$$
S=\frac{1}{4} \frac{A}{\ell_{\mathrm{Pl}}^{2}},
$$

где $\ell_{\mathrm{Pl}}=\sqrt{\hbar G / c^{3}} \sim 10^{-33} \mathrm{cм}-$ планковская длина $(\hbar-$ постоянная Планка, $c-$ скорость света). Мы будем пользоваться системой единиц, в которой $\hbar=c=1$, поэтому $\ell_{\mathrm{Pl}}=\sqrt{G}$, а планковская масса равна $m_{\mathrm{Pl}}=\sqrt{\hbar c / G}=1 / \sqrt{G} \sim 10^{-5}$ г.

Природа излучения Хокинга и его чернотельного спектра заключается в нетривиальной причинной структуре пространства-времени, содержащего черную дыру. Решающую роль играет существование горизонта событий. То же самое имеет место в пространстве-времени Риндлера, которое получается преобразованием двумерного плоского пространства-времени Минковского от “обычных" координат $(t, x)$ и метрики $d s^{2}=d t^{2}-d x^{2}$, привязанных к совокупности инерциальных наблюдателей, к так называемым риндлеровым координатам $(\eta, \xi)\left(t=(1 / a) e^{a \xi} \operatorname{sh} a \eta\right.$, $\left.x= \pm(1 / a) e^{a \xi} \operatorname{ch} a \eta,-\infty<\eta<\infty,-\infty<\xi<\infty\right)$ и метрике $d s^{2}=e^{2 a \xi}\left(d \eta^{2}-d \xi^{2}\right)$. Таким образом, пространство-время Риндлера статичное и локально-плоское, но отличается от двумерного пространства-времени Минковского глобальной структурой, так как оно покрывает лишь половину последнего и, кроме того, обладает горизонтом событий $t= \pm x(\eta= \pm \infty, \xi=$ const). Риндлеровские наблюдатели $\xi=$ const постоянно ускоренные. Норма вектора ускорения $a^{\mu}$ равна $\alpha=\sqrt{-a^{\mu} a_{\mu}}=a e^{-a \xi}$. Изучая квантовую теорию поля в пространстве-времени Риндлера, Унру [10] обнаружил конечнотемпературную квантовую теорию поля с температурой

$$
T_{\mathrm{U}}=\frac{a}{2 \pi} .
$$

Видно,что температура пропорциональна ускорению риндлеровского наблюдателя, сидящего в точке $\xi=0$, для которого $g_{00}=1$. Однако все наблюдатели эквивалентны (мы всегда можем сдвинуть пространственную координату $\xi \rightarrow \xi-\xi_{0}$ ). Температура не является инвариантом: это временна́я компонента вектора теплового потока. Последнее означает, что каждый наблюдатель измеряет температуру Унру, если пользуется своим собственным временем $\tau(d s=d \tau)$. Если тот же наблюдатель пользуется локальными часами, показывающими локальное координатное время $t$ $\left(d s=\sqrt{g_{00}} d t\right)$, то измеряемая им локальная температура равна

$$
T_{\mathrm{loc}}=\frac{T_{\mathrm{U}}}{\sqrt{g_{00}}}=\frac{a}{2 \pi} e^{-a \xi}=\frac{\alpha}{2 \pi}
$$

что пропорционально локальному ускорению наблюдателя $\alpha$. Сам факт, что постоянно ускоренный наблюдатель (детектор) будет детектировать реальные частицы в вакууме, был известен людям, занимающимся квантовой электродинамикой, давно. Этот эффект объяснялся как результат изменения вакуумного состояния под воздействием внешних сил, вызывающих ускорение. То же самое происходит в пространстве-времени с горизонтами событий. Но тот факт, что спектр является тепловым, оказался совершенно новым и чисто релятивистским явлением. Мы знаем из университетского курса термодинамики, что условием теплового равновесия в статическом пространстве-времени является выполнение следующего соотношения: $T_{\text {loc }} \sqrt{g_{00}}=$ const. Таким образом, все риндлеровские наблюдатели находятся в тепловом равновесии друг с другом. Является ли пространство Риндлера в этом 
смысле единственным? Чтобы ответить на этот вопрос, рассмотрим двумерное статическое пространство-время общего вида с метрикой

$$
d s^{2}=e^{\nu} d t^{2}-d \rho^{2}=e^{\nu} d t^{2}-e^{\lambda} d q^{2} .
$$

В риндлеровом случае $\rho=(1 / a) e^{a \xi}, e^{\nu}=a^{2} \rho^{2}=g_{00}$. Статический наблюдатель имеет постоянное ускорение, инвариант которого равен

$$
\alpha=\frac{1}{2}\left|\frac{d \nu}{d \rho}\right|=\frac{1}{2}\left|\frac{d \nu}{d q}\right| e^{-\lambda / 2},
$$

при этом (теперь локальный) параметр Риндлера $\varkappa$, который называется “поверхностной гравитацией", равен

$$
\varkappa=\frac{1}{2}\left|\frac{d \nu}{d q}\right| e^{(\nu-\lambda) / 2}=\frac{1}{2}\left|\frac{d \nu}{d \rho}\right| e^{\nu / 2} .
$$

Условие теплового равновесия требует, чтобы $\varkappa=$ const, следовательно, $g_{00}=C \rho^{2}$, и это доказывает, что пространство-время Риндлера - единственное, в котором статические наблюдатели находятся в тепловом равновесии.

В силу принципа эквивалентности Эйнштейна мы можем все, что узнали, изучая пространство-время Риндлера, перенести на статические гравитационные поля, в особенности на сферически-симметричные, поскольку после фиксирования сферических углов $\theta$ и $\varphi$ последние фактически превращаются в двумерные псевдоевклидовы поверхности. Разумеется, в общем случае эти поверхности искривленные, и принцип эквивалентности справедлив лишь локально, а статические наблюдатели не будут уже в тепловом равновесии друг с другом. Такая температура Унру зависит от наблюдателя и не может рассматриваться как внутреннее свойство данного пространства-времени. Однако для многообразий, содержащих черную дыру, положение горизонта является абсолютным и не зависит от наблюдателя, так что температура действительно служит характеристикой самого пространства-времени. Чтобы узнать значение этой температуры, достаточно вычислить значение поверхностной гравитации на горизонте событий $\varkappa_{\mathrm{H}}$. Для черной дыры Шварцшильда со знаменитой метрикой

$$
d s^{2}=F d t^{2}-\frac{1}{F} d r^{2}-r^{2}\left(d \theta^{2}+\sin ^{2} \theta d \varphi^{2}\right), \quad F=1-\frac{2 G m}{r},
$$

где $r$ - радиус сферы (в том смысле, что ее площадь равна $4 \pi r^{2}$ ), горизонт событий расположен на так называемом гравитационном радиусе $r_{\mathrm{g}}=2 G m$, где поверхностная гравитация равна

$$
\varkappa_{\mathrm{H}}=\frac{1}{2}\left|\frac{d \nu}{d r}\right| e^{(\nu-\lambda) / 2}=\frac{1}{2} F^{\prime}\left(r_{\mathrm{H}}\right)=\left.\frac{G m}{r^{2}}\right|_{r_{\mathrm{g}}}=\frac{1}{4 G m}
$$

Таким образом, температура Хокинга есть как раз температура Унру на горизонте событий, измеряемая удаленным наблюдателем на бесконечности. То же справедливо и для черных дыр Керра-Ньюмена. Отметим, что вне горизонта событий $r>r_{\mathrm{g}}$ шварцшильдовы наблюдатели не находятся в тепловом равновесии друг с другом, и 
это является термодинамическим обоснованием существования излучения Хокинга и, таким образом, испарения черных дыр. Следует подчеркнуть, что и температура черной дыры, и ее энтропия являются глобальными характеристиками, потому что само их появление обязано существованию горизонта событий.

Испаряясь, черные дыры становятся все меньше и меньше, достигая в конце концов планковских размеров, где существенную роль должна играть все еще неизвестная квантовая теория гравитации. Так как излучение квантовано, масса черной дыры также должна быть квантованной. Конечно, соотношение не является столь прямолинейным, так как черная дыра не обязательно переходит в черную же дыру при единичном акте излучения, но новая черная дыра неизбежно образуется опять же в процессе излучения. В массу черной дыры дают вклад не только массы покоя и кинетические энергии (включая угловые моменты) частиц, но также кулоновская энергия их электрических и калибровочных зарядов, магнитная энергия токов и все другие физические поля, заключенные за горизонтом событий. Но общим свойством для всех типов черных дыр является универсальное соотношение (2) для площади горизонта. Следовательно, квантование черной дыры означает квантование ее энтропии. Более того, само термодинамическое описание возможно, только если скачок энтропии (вследствие квантованности массы, заряда и углового момента) в процессе испарения черной дыры пренебрежимо мал по сравнению с абсолютными значениями параметров, в то время как понятие энтропии как меры информации, недоступной или игнорируемой, все еще работает. Именно эта особенность энтропии привела к общему мнению, что квазиклассическое квантование черных дыр может пролить свет на структуру будущей полной квантовой теории гравитации или, по крайней мере, снабдит нас некоторыми правилами отбора в попытках построить такую теорию. Квантование черной дыры как целостного объекта было предложено Бекенштейном много лет назад [11]. Идея базируется на замечательном наблюдении, что площадь горизонта неэкстремальной черной дыры является адиабатическим инвариантом. В этом случае правило квантования Бора-Зоммерфельда предсказывает эквидистантый спектр для площади горизонта и, следовательно, для энтропии черной дыры. Мысленный эксперимент показывает, что вследствие квантовых эффектов минимальное увеличение площади горизонта в результате захвата нейтральной или электрически заряженной частицы примерно равно $\Delta A_{\min } \approx 4 \ell_{\mathrm{Pl}}^{2}$. Это предполагает, что для энтропии черной дыры

$$
S_{\mathrm{BH}}=\gamma_{0} N, \quad N=1,2, \ldots,
$$

где $\gamma_{0}$ - численный коэффициент порядка единицы. В своей знаменитой работе по спектроскопии черных дыр Бекенштейн и Муханов [12] связали значение энтропии черной дыры с числом $g_{n}$ микросостояний, соответствующих конкретному внешнему макросостоянию, посредством хорошо известной из статистической физики формулы $g_{n}=e^{S_{\mathrm{BH}}(n)}$, т. е. $g_{n}-$ степень вырожденности $n$-го собственного значения площади. Так как $g_{n}$ должно быть целым числом, авторы заключили, что

$$
\gamma_{0}=\ln k, \quad k=2,3, \ldots
$$

В свете теории информации и провозглашенного Уилером принципа "It from Bit" значение $\ln 2$ кажется наиболее подходящим. 
Логарифмическое поведение кванта энтропии $\gamma_{0}$ следует также из петлевой квантовой гравитации. Было показано [13], [14], что энтропия шварцшильдовой черной дыры пропорциональна площади горизонта и некоторому численному коэффициенту, который называется параметром Иммирзи. Для того чтобы выполнялось соотношение Бекенштейна-Хокинга (2) и получалось одно из возможных значений для $\gamma_{0}(9)$, этот параметр должен быть равным $\ln 2 /(\pi \sqrt{3})$, если фундаментальной группой в основе петлевой квантовой гравитации является группа $S U(2)$, и равным $\ln 3 /(2 \pi \sqrt{2})$, если это группа $S U(3)$. Выбор значения $\gamma_{0}$ приводит к минимально возможному изменению массы черной дыры. Ход [15], воспользовавшись принципом соответствия Бора, заключил, что это $\ln 3$, заметив, что

$$
G m \operatorname{Re} w=0.0437123=\frac{\ln 3}{8 \pi} .
$$

Значение $\gamma_{0}$, так же как значение параметра Иммирзи и, следовательно, выбор фундаментальной группы в петлевой квантовой гравитации, должно быть универсальным. Поэтому неудивительно, что начались поиски аналитических методов вычисления квазинормальных частот для различных типов черных дыр. Используя довольно изощренные методы общей теории обыкновенных дифференциальных уравнений, Мольт и Нейтцке показали [16], [17], что для скалярных и тензорных возмущений вокруг шварцшильдовых черных дыр значение $\ln 3$ является точным. Для черных дыр более общего вида соответствующие вычисления были выполнены в работе [18]. Оказалось, что простое значение $\ln 3$ для коэффициента $\gamma_{0}$ ни в коем случае не является универсальным, а, наоборот, исключительным. Именно поэтому мы используем выражение "загадка $\ln 3$ ".

В нижеследующих разделах мы построим модель, которая, хотя и не является реальной черной дырой, обладает ее основными качествами, а именно в ней есть горизонт событий - но локальный, и температура - но тоже локальная. Затем мы построим локальную термодинамику для такой модели и покажем, как “загадка ln 3" может быть разгадана. Есть надежда, что наша модель поможет в понимании скрытой физики многих других интересных свойств квазиклассических черных дыр.

\section{2. “СТАНДАРТНАЯ МОДЕЛЬ"}

2.1. Квантовые тонкие оболочки. Построение нашей модели мы начнем с краткого описания конкретной частной модели квантовой черной дыры Шварцшильда, а именно с теории квантованных сферически-симметричных тонких пылевых оболочек [19], [20] - простейшего обобщения точечной частицы. В этом случае имеется только одна динамическая степень свободы - радиус оболочки (реальные гравитоны отсутствуют в силу сферической симметрии по теореме Биркгоффа), и квантово-гравитационное функциональное уравнение Уилера-де Витта сводится к стационарному одномерному уравнению Шредингера, но не дифференциальному, а в конечных разностях. Самым важным является тот факт, что такая модель самосогласована, в ней учтено обратное влияние гравитирующего источника (тонкой оболочки) на геометрию полного многообразия Шварцшильда, имеющего нетривиальную причинную структуру. Геодезически полное пространство-время Шварцшильда имеет геометрию непроходимой черной дыры (ее еще называют вечной 
черной дырой). Имеются две асимптотически плоские области с пространственными бесконечностями, которые связаны так называемым мостом Эйнштейна-Розена (горловиной). Две стороны моста причинно не связаны и отделены друг от друга горизонтами событий прошлого и будущего. Внутри оболочки мы имеем часть метрики Шварцшильда с массовым параметром $m_{\text {in }}$, тогда как шварцшильдова масса снаружи равна $m_{\text {out }}$.

В квантовой механике нет траекторий, и волновая функция оболочки "чувствует" существование горизонта и обеих бесконечностей. В результате возникает необходимость наложения дополнительного граничного условия, и появляются два квантовых числа для двух величин, описывающих квантовые состояния (при фиксированном $\left.m_{\text {in }}\right),-$ "голой” массы оболочки $M$ (суммы масс всех составляющих) и их полной массы (энергии) $\Delta m=m_{\text {out }}-m_{\text {in }}$, которая включает гравитационный дефект масс. Дискретный спектр масс связанных состояний имеет следующий вид ( $n$ и $p$ - целые числа):

$$
\begin{aligned}
\frac{2(\Delta m)^{2}-M^{2}}{\sqrt{M^{2}-(\Delta m)^{2}}} & =\frac{2 m_{\mathrm{Pl}}^{2}}{\Delta m+2 m_{\mathrm{in}}} n, \\
M^{2}-(\Delta m)^{2} & =2(1+2 p) m_{\mathrm{Pl}}^{2} .
\end{aligned}
$$

Если задана голая масса $M$, то изменение квантового состояния вызывает изменение как массы внутри оболочки $m_{\text {in }}$, так и полной массы системы $m_{\text {out }}$. Следовательно, в процессе гравитационного коллапса полная масса уменьшается вследствие излучения, а внутренняя масса растет (в нашем случае - за счет рождения новых оболочек). Когда такой процесс может остановиться? Естественным пределом является пересечение моста Эйнштейна-Розена, поскольку такой переход требует (по крайней мере в квазиклассическом режиме) включения в геометрию бесконечно большого объема, вероятность чего, конечно же, равна нулю. Компьютерное моделирование показало, что процесс квантового коллапса наших оболочек останавливается, когда главное квантовое число $n=0$ становится равным нулю.

Точка $n=0$ в нашем спектре - очень особая. В этом случае оболочка не "чувствует" не только внешней области (что естественно для сферически-симметричной конфигурации), но она ничего не знает о том, что делается внутри. Она "чувствует" лишь саму себя. Такая ситуация напоминает свойство классических черных дыр "не иметь волос". Наконец, когда все оболочки (и первоначальная, и новорожденные) окажутся в соответствующих состояниях $n_{i}=0$, вся система целиком не будет "помнить" своей собственной истории. Тогда именно такое состояние "беспамятства" может быть названо "квантовая черная дыра". Отметим, что полные массы всех оболочек удовлетворяют соотношению $\Delta m_{i}=(1 / \sqrt{2}) M_{i}$.

2.2. Классический аналог квантовой черной дыры Шварцшильда. Конечное состояние при квантовом гравитационном коллапсе можно рассматривать как некоторое стационарное распределение материи. Таким образом, можно надеяться, что для достаточно массивной черной дыры такое распределение описывается приближенно как классическая статическая сферически-симметричная идеальная жидкость с плотностью энергии $\varepsilon$ и (эффективным) давлением $p$, и при этом выполняются классические уравнения Эйнштейна. Это как раз то, что мы называем классическим аналогом квантовой черной дыры. Разумеется, такое распределение 
должно быть очень специфическим. Чтобы изучить его свойства, рассмотрим ситуацию детальнее.

Любая статическая сферически-симметричная метрика может быть записана в виде

$$
d s^{2}=e^{\nu} d t^{2}-e^{\lambda} d r^{2}-r^{2}\left(d \theta^{2}+\sin ^{2} \theta d \varphi^{2}\right) .
$$

Здесь $r$ - радиус сферы с площадью $A=4 \pi r^{2}, \nu=\nu(r), \lambda=\lambda(r)$. У нас имеется только три (статических и сферически-симметричных) уравнения Эйнштейна. Уравнение связи может быть представлено в интегральной форме:

$$
e^{-\lambda}=1-\frac{2 G m(r)}{r}
$$

где

$$
m(r)=4 \pi \int_{0}^{r} \varepsilon \tilde{r}^{2} d \tilde{r}
$$

- массовая функция, которую следует идентифицировать как $m_{\text {in }}$. Теперь принцип "беспамятства" явным образом формулируется как требование $m(r)=a r$, т. е.

$$
e^{-\lambda}=1-2 G a=\mathrm{const}, \quad \varepsilon=\frac{a}{4 \pi r^{2}} .
$$

Мы можем также ввести функцию голой массы $M(r)$ (массы системы внутри сферы радиуса $r$ за вычетом гравитационного дефекта масс):

$$
M(r)=\int \varepsilon d V=4 \pi \int_{0}^{r} \varepsilon e^{\lambda / 2} \tilde{r}^{2} d \tilde{r}=\frac{a r}{\sqrt{1-2 G a}} .
$$

Оставшиеся два уравнения теперь могут быть разрешены относительно $p(r)$ и $e^{\nu}(r)$. Общее решение довольно сложное и громоздкое, но правильный нерелятивистский предел для давления $p(r)$ (мы обязаны воспроизвести известное уравнение гидростатического равновесия) дает только следующее частное решение:

$$
p(r)=\frac{b}{4 \pi r^{2}}, \quad b=\frac{1}{G}(1-3 G a-\sqrt{1-2 G a} \sqrt{1-4 G a}) .
$$

Очевидно, что решение существует только в случае $a \leqslant 1 /(4 G)$, и тогда $b \leqslant a$. Физический смысл этих неравенств заключается в том, что скорость звука не может превышать скорость света: $v_{\text {sound }}^{2}=(b / a) \leqslant 1=c^{2}$, причем равенство достигается как раз при $a=b=1 /(4 G)$. Наконец, для временно́й компоненты метрического тензора $g_{00}=e^{\nu}$ имеем

$$
e^{\nu}=C_{0}^{2} r^{4 b /(a+b)}=C_{0}^{2} r^{2 G(a+b) /(1-2 G a)} .
$$

Таким образом, требование свойства "беспамятства" и существования правильного нерелятивистского предела позволило нам получить двухпараметрическое семейство статических решений (вторым параметром является значение граничного радиуса распределения). Но нам необходимо иметь однопараметрическое семейство, поэтому продолжим исследование.

Очевидно, что точка $r=0$ является сингулярной как для распределения материи, так и для метрического коэффициента $g_{00}$. Чтобы выяснить, с какого типа сингулярностью мы имеем дело, вычислим тензор кривизны Римана. Оказывается, что при 
$b<a$ этот тензор действительно расходится при $r=0$. Но если $a=b=1 /(4 G)$, то мы сталкиваемся с чудесным явлением - расходящиеся (до сих пор) компоненты оказываются равными нулю. Таким образом, требуя дополнительно к двум предыдущим очень естественным условиям третье (тоже естественное), а именно отсутствие реальной физической сингулярности кривизны при $r=0$, мы получаем следующее однопараметрическое семейство решений уравнений Эйнштейна:

$$
e^{\nu}=C_{0}^{2} r^{2}, \quad e^{\lambda}=2, \quad \varepsilon=p=\frac{1}{16 \pi G r^{2}} .
$$

Оказывается, уравнение состояния нашей идеальной жидкости наиболее жесткое из возможных. Постоянная интегрирования $C_{0}$ может быть определена из сшивки внутренней и внешней метрик на некоторой границе радиуса $r=r_{0}$. Предположим, что при $r>r_{0}$ пространство-время пустое, так что внутреннее решение должно быть сшито с метрикой Шварцшильда с некоторой массой $m$. Разумеется, для компенсации скачка давления $\Delta p\left(=p\left(r_{0}\right)=p_{0}\right)$ нужно включить в нашу модель поверхностное натяжение $\Sigma$, так что фактически мы имеем дело с некоторой жидкостью. Легко проверить, что

$$
\begin{gathered}
C_{0}^{2}=\frac{1}{2 r_{0}^{2}}, \quad \Delta p=\frac{2 \Sigma}{\sqrt{2} r_{0}}, \quad e^{\nu}=\frac{1}{2}\left(\frac{r}{r_{0}}\right)^{2}, \\
p_{0}=\varepsilon_{0}=\frac{1}{16 \pi G r_{0}^{2}}, \quad m=m_{0}=\frac{r_{0}}{4 G} .
\end{gathered}
$$

Отметим, что голая масса $M=\sqrt{2} m$, т. е. соотношение в точности такое же, как и для квантовой оболочки в состоянии “беспамятства". Кроме того, $r_{0}=4 G m_{0}$, так что размеры нашей аналоговой модели в два раза больше, чем у классической черной дыры той же массы.

Особая точка нашего решения $r=0$ - не просто тривиальная координатная сингулярность, подобная такой же в трехмерном сферически-симметричном пространстве, потому что теперь $d s^{2}(r=0)=0$. Это выглядит как горизонт событий. И действительно, двумерное $(t-r)$-сечение нашей метрики описывает локально-плоское многообразие. Так как статические наблюдатели $r=$ const являются фактически постоянно ускоренными, это есть пространство-время Риндлера с горизонтом событий при $r=0$. Соответствующий параметр Риндлера, в более общем случае называемый “поверхностной гравитацией”, равен

$$
\varkappa=\frac{1}{2}\left|\frac{d \nu}{d r}\right| e^{(\nu-\lambda) / 2}=\frac{C_{0}}{\sqrt{2}}=\frac{1}{2 r_{0}} .
$$

Следовательно, температура Унру в нашей модели равна $T_{\mathrm{U}}=1 /\left(4 \pi r_{0}\right)=1 /(16 \pi G m)$, что в два раза меньше температуры Хокинга для черной дыры Шварцшильда:

$$
T_{\mathrm{H}}=\frac{1}{8 \pi G m}=2 T_{\mathrm{U}}
$$

Подведем итог. Построена чисто классическая модель, обладающая некоторыми свойствами (квази)классических черных дыр: есть горизонт событий и появилась 
температура, но эти свойства уже локальные, а не глобальные. Действительно, поверхность $r=0$ не может быть пересечена по определению, поэтому горизонт событий в нашей модели локальный. Температура также локальная: $T_{\mathrm{loc}}=T_{\mathrm{U}} / \sqrt{g_{00}}=$ $1 /(2 \sqrt{2} \pi r)$, и не зависит от граничного значения радиуса $r_{0}$. Имеется еще одна важная особенность: если убрать внешний слой некоторой толщины, ничто внутри не изменится. Это является отражением того факта, что все части нашего распределения материи находятся в тепловом равновесии.

Квантовая природа излучения и тот факт, что энтропия черной дыры имеет эквидистантный спектр, подсказывают, что наше распределение состоит на самом деле из некоторого числа квазичастиц, "гравитационных фононов". Таким образом, имея локальные интенсивные параметры - эффективное давление $p(r)$, температуру $T_{\text {loc }}(r)$, химический потенциал $\mu(r)$, и экстенсивные параметры $-M$, объем $V$, энтропию $S$ и число “частиц" $N$, мы уже готовы к построению локальной термодинамики.

2.3. Термодинамика. Первый закон термодинамики гласит:

$$
d M=\varepsilon d V=T_{\mathrm{loc}} d S-p d V+\mu d N .
$$

Поделив левую и правую части этого выражения на элемент объема $d V$, получаем первый закон в локальной записи:

$$
\varepsilon(r)=T_{\mathrm{loc}}(r) s(r)-p(r)+\mu(r) n(r),
$$

где $s$ и $n$ - плотность энтропии и плотность числа частиц соответственно. В нашей модели $\varepsilon=p$, а что можно сказать об $s ?$ Локальный наблюдатель не в состоянии вычислить плотность энтропии без знания соответствующей микроскопической структуры, но он может задать вопрос своему глобальному собрату, сидящему на бесконечности и достаточно образованному (прочитал нужные книги), чтобы знать, что полная энтропия черной дыры равна $S=(1 / 4 G) A_{\mathrm{hor}}$, что для Шварцшильда $\left(A_{\text {hor }}=4 \pi r_{\mathrm{g}}^{2}\right)$ дает

$$
S=\frac{\pi}{G} r_{\mathrm{g}}^{2}=\frac{\pi r_{0}^{2}}{4 G} .
$$

Получив такую информацию, локальный наблюдатель может сделать вывод, что

$$
s(r)=\frac{1}{8 \sqrt{2} G r}, \quad T_{\mathrm{loc}}(r) s(r)=\frac{1}{32 \pi G r^{2}} .
$$

Вспоминая теперь, что $\varepsilon=1 /\left(16 \pi G r^{2}\right)$, получаем

$$
T_{\mathrm{loc}}(r) s(r)=\frac{1}{2} \varepsilon, \quad \mu(r) n(r)=\frac{3}{2} \varepsilon .
$$

Нам еще понадобится выражение для свободной энергии $F$ :

$$
F=\int f d V, \quad f=\varepsilon-T_{\mathrm{loc}} s=\frac{1}{2} \varepsilon .
$$

Известно, что условие теплового равновесия для системы в статическом гравитационном поле имеет вид

$$
T \sqrt{g_{00}}=\text { const }, \quad \mu \sqrt{g_{00}}=\text { const } .
$$


Константы в правых частях являются универсальными в нашей модели - они не зависят от значения граничного радиуса $r_{0}$. Следовательно, их отношение - тоже универсальная константа. Поэтому имеем

$$
\frac{\mu}{T}=3 \frac{s}{n}=3 \frac{S}{N}=3 \gamma_{0} .
$$

Мы видим теперь, что энтропия естественным образом квантуется:

$$
S=\gamma_{0} N, \quad N=1,2, \ldots .
$$

2.4. Разрешение “загадки $\ln 3$ ”. Чтобы вычислить коэффициент $\gamma_{0}-$ квант энтропии, мы должны сделать некоторые предположения о микроскопической структуре нашей модели. Предположим, что внутри распределение материи состоит из $N$ чернодырных фононов с эквидистантным спектром возбуждений:

$$
\varepsilon_{n}=\omega n, \quad n=1,2, \ldots
$$

В таком случае функция распределения полной системы есть произведение функций распределения для каждого фонона:

$$
Z_{\text {tot }}=\left(Z_{1}\right)^{N}, \quad Z_{1}=\sum_{n} e^{-\varepsilon_{n} / T}=\sum_{n}\left(e^{-\omega / T}\right)^{n}=\frac{e^{-\omega / T}}{1-e^{-\omega / T}} .
$$

Будет естественным предположить, что частота $\omega$ как раз и является резонансной частотой черной дыры - существование таковой следует, как уже было разъяснено выше, из свойств квазинормальных мод. Частота $\omega$ есть временна́я компонента четырехвектора, но то же самое относится и к температуре $T$, так что их отношение не зависит от того, какими часами пользуется локальный наблюдатель. Пусть наблюдатель использует свое собственное время, тогда $T$ - температура Унру $T_{\mathrm{U}}$, которая постоянна по всему распределению материи. Функция распределения является инвариантом, и мы можем вычислить ее и другим способом, используя термодинамические соотношения. Действительно, рассмотрим некоторый малый элемент объема $d V$ и функцию распределения в нем $Z_{\text {small }}$. Тогда, воспользовавшись хорошо известной формулой для свободной энергии $F=-T \ln Z$, напишем ее для элемента объема:

$$
d F=f d V=-T_{\mathrm{loc}} \ln Z_{\mathrm{small}},
$$

где, как и ранее, мы использовали локальные значения термодинамических величин. Отсюда получаем

$$
\int \frac{f}{T_{\mathrm{loc}}} d V=-\sum \ln Z_{\mathrm{small}}=-\ln Z_{\mathrm{tot}} .
$$

Левая часть этого выражения равна

$$
\int \frac{f}{T_{\mathrm{loc}}} d V=\frac{1}{2} \int \frac{\varepsilon}{T_{\mathrm{loc}}}=\frac{\pi r_{0}^{2}}{4 G}=\frac{\pi r_{\mathrm{g}}^{2}}{G}=S,
$$

где $r_{\mathrm{g}}$ - радиус Шварцшильда, а $S$ - полная энтропия черной дыры. В результате получаем важное соотношение

$$
e^{-S}=Z_{\text {tot }}=\left(Z_{1}\right)^{N},
$$


из которого следует, что

$$
\frac{e^{-\omega / T}}{1-e^{-\omega / T}}=e^{-S / N}=e^{-\gamma_{0}}, \quad e^{\gamma_{0}}=e^{\omega / T}-1 .
$$

Чтобы продвинуться дальше, рассмотрим необратимый процесс превращения массы (энергии) системы в излучение с точки зрения термодинамики. В нашей модели такой процесс происходит как раз на границе $r=r_{0}$, а тонкая оболочка с нулевой плотностью энергии и поверхностным натяжением $\Sigma$ служит преобразователем, снабжающим излучение добавочной энергией и добавочной энтропией, что напоминает модель "кирпичной стены". Природа рассматриваемого излучения квантовая, так как классически наша система ничего не излучает. Скачок в температуре Унру для внутренних и внешних околограничных наблюдателей в точности компенсируется гравитационным полем, создаваемым поверхностным натяжением. Представим себе, что пограничный слой толщины $\Delta r_{0}$ преобразуется в излучение, уменьшая тем самым размер внутренней области до радиуса $\left(r_{0}-\Delta r_{0}\right)$. Его энергия (масса) равна $\Delta M=\varepsilon \Delta V$. K ней мы должны прибавить энергию, высвободившуюся при сдвиге в результате работы сил поверхностного натяжения, которая в точности равна $\sum d\left(4 \pi r_{0}^{2}\right)=p d \Delta V=\varepsilon \Delta V=\Delta M$. Таким образом, и энергия, и энтропия в конвертере в два раза выше, чем в любом внутреннем слое той же толщины. И эта двойная энергия передается излучаемым квантам. Ясно, что их частота и температура удваиваются, так что

$$
\frac{\operatorname{Re} w}{T_{\mathrm{H}}}=\frac{\omega}{T_{\mathrm{U}}}=\ln 3,
$$

как следует из вида спектра квазинормальных мод для шварцшильдовых черных дыр. Подставляя это в уравнение (34) и вспоминая, что $3-1=2$, получаем

$$
\gamma_{0}=\ln 2 .
$$

Поскольку излучаемая энергия термализована, интерпретация равенства $d m$ и $\operatorname{Re} w$ некорректна. Это и разрешает “загадку $\ln 3$ ”.

\section{3. ЗА РАМКАМИ “СТАНДАРТНОЙ МОДЕЛИ"}

Предложенная выше модель очень жесткая. Поэтому возникает вопрос: какое из наложенных условий может быть ослаблено? Напомним шаги, приведшие к конечному результату. Во-первых, мы потребовали выполнения условия "беспамятства". Это было необходимо для обеспечения мимикрии черных дыр. Во-вторых, мы предположили, что тензор энергии-импульса соответствует идеальной жидкости. Тогда требование отсутствия сингулярности кривизны на нулевом радиусе привело и к появлению температуры, и к единственно возможному (наиболее жесткому) уравнению состояния. Разумеется, тепловое равновесие - решающее свойство нашей модели, но как насчет изотропии давления в жидкости?

Чтобы прояснить этот момент, рассмотрим общий вид статической сферически-симметричной метрики, в которой все статические наблюдатели находятся во взаимном тепловом равновесии. Как мы уже знаем, пространство-время в этом случае должно быть прямым произведением (локально-плоского) многообразия Риндлера на двумерную сферу радиуса $R$ :

$$
d s^{2}=a^{2} \rho^{2} d t^{2}-d \rho^{2}-R^{2}(\rho)\left(d \theta^{2}+\sin ^{2} \theta d \varphi^{2}\right),
$$


где $a$ - параметр ускорения, а $R(\rho)$ - единственная неизвестная функция радиальной координаты $\rho$. Уравнения Эйнштейна теперь принимают следующий вид:

$$
\begin{aligned}
-\frac{2 R^{\prime \prime}}{R}+\frac{1-R^{\prime 2}}{R^{2}} & =8 \pi G \varepsilon, \\
-2 \frac{R^{\prime}}{\rho R}+\frac{1-R^{\prime 2}}{R^{2}} & =-8 \pi G p_{\mathrm{r}}, \\
-\frac{R^{\prime \prime}}{R}-\frac{R^{\prime}}{\rho R} & =-8 \pi G p_{\mathrm{t}} .
\end{aligned}
$$

Штрих означает обычную производную, и мы предположим, что, вообще говоря, радиальное давление $p_{\mathrm{r}}$ не равно тангенциальному давлению $p_{\mathrm{t}}$. C наложением условия "беспамятства" $R^{\prime}=\alpha=$ const вышеприведенные уравнения становятся алгебраическими; кроме того, в этом случае $\varepsilon+p_{\mathrm{r}}=2 p_{\mathrm{t}}$ и при изотропном давлении $p_{\mathrm{r}}=p_{\mathrm{t}}$ мы возвращаемся к предыдущему результату. Вспомним теперь, что соотношение между голой и полной массами, а именно $M=\sqrt{2} m$, оказалось в нашей модели таким же, как и для квантованных тонких пылевых оболочек в состоянии "беспамятства". Этот факт указывает на то, что наш классический аналог состоит целиком из массивных компонент, но в реальности внутри классических черных дыр может быть и излучение (т. е. безмассовые частицы). Рассмотрим теперь экстремальную ситуацию, когда классический аналог состоит только из безмассовых компонент. Тогда $\varepsilon=p_{\mathrm{r}}+2 p_{\mathrm{t}}$ и, следовательно, $p_{\mathrm{r}}=0, \varepsilon=2 p_{\mathrm{t}}, \alpha=1 / \sqrt{3}$. Такое странное уравнение состояния означает, что мы имеем дело не с конденсированной материей, а скорее с набором тонких оболочек с (исчезающе) малыми энергиями, которые составлены из безмассовых частиц, вращающихся по сферам постоянного радиуса во всех возможных направлениях [21]. Но такое распределение нестабильно, поскольку эти орбиты совпадают с последней (неустойчивой) световой орбитой во внешней метрике Шварцшильда. В промежуточном случае мы получаем смесь двух экстремальных моделей, и орбиты становятся устойчивыми (пограничные орбиты устойчивы благодаря ненулевому поверхностному натяжению). Более того, если предположить, что эти два типа распределения материи не взаимодействуют (иначе как гравитационно), что кажется совершенно естественным в духе нашего условия "беспамятства", то несложно показать с помощью раздельных уравнений неразрывности, что $R^{\prime}=$ const, а часть смеси, представляющая идеальную жидкость, имеет максимально жесткое уравнение состояния.

Такая обобщенная модель обладает вполне достойными свойствами. Во-первых, значение $R^{\prime}$ уже не определено столь жестко, вместо этого мы имеем $1 / 3<\alpha^{2} \leqslant 1 / 2$. Во-вторых, эти вращающиеся по орбитам безмассовые частицы могут рассматриваться как остатки излученных квазинормальных мод и в то же время как первопричина эквидистантного "фононного" спектра в идеальной жидкости. В-третьих, "хокинговское испарение" в нашей аналоговой модели можно теперь рассматривать как индуцированное излучение, посредством туннелирования прошедшее сквозь барьер, поставленный поверхностным натяжением на границе. В-четвертых, появляется возможность построения следующей модели: представим себе, что полное пространство-время состоит из трех совершенно различных областей - внутренней, внешней и средней, причем во внутренней части мы имеем нашу классическую аналоговую модель плюс вышеописанную безмассовую составляющую, во внешней об- 
ласти - вакуумное решение Шварцшильда, а область между ними заполним чистым излучением с равным нулю следом тензора энергии-импульса. Предположим, что по отдельности внутренняя и внешняя части находятся в тепловом равновесии, но при разных температурах, поскольку они разделены тонкой оболочкой с ненулевым натяжением, поддерживающей температурный градиент. Выясняется, что очевидное, казалось бы, изотропное уравнение состояние $\varepsilon=3 p$ в средней области не удовлетворяет одновременно двум требованиям - поддержания теплового равновесия и удовлетворения статическим уравнениям Эйнштейна, так что это не привычное чернотельное равновесное излучение, а скорее множество сферически-симметричных тонких оболочек с орбитальными безмассовыми составляющими, которые путешествуют между внутренней и внешней границами. Представим теперь, что мы двигаем границу между средней и внешней областями, держа фиксированной шварцшильдову массу полной системы. Положение границы между средней и внутренней областями будет определяться, скажем, принципом максимума энтропии. Что при этом будет происходить, неизвестно, но перспективы очень интересны, потому что состояние всей системы зависит теперь от двух параметров: полной массы (энергии) и положения внешней границы, в отличие от классической черной дыры Шварцшильда. Кроме того, в результате решение будет самосогласованным, т. е. будет учитывать обратное влияние распределения материи на метрику. Поэтому можно ожидать даже появления некоего экстремального состояния с нулевой температурой, как в случае черной дыры Рейсснера-Нордстрема.

Мы еще не знаем, как использовать термодинамическое соотношение в столь сложной системе, но работа над этим уже ведется.

Благодарности. Работа была выполнена при финансовой поддержке РФФИ (грант № 10-02-00635_а).

\section{Список литературы}

[1] S. W. Hawking, G. F. R. Ellis, The Large Scale Structure of Space-Time, Cambridge Monographs of Mathematical Physics, 1, Cambridge Univ. Press, London, 1973.

[2] R. Ruffini, J. A. Wheeler, Phys. Today, 24:1 (1971), 30-36.

[3] T. Regge, J. A. Wheeler, Phys. Rev., 108:4 (1957), 1063-1069.

[4] S. Chandrasekhar, The Mathematical Theory of Black Holes, International Series of Monographs on Physics, 69, Oxford Univ. Press, New York, 1983.

[5] D. Cristodoulou, Phys. Rev. Lett., 25:22 (1970), 1596-1597.

[6] D. Cristodoulou, R. Ruffini, Phys. Rev. D, 4:12 (1971), 3552-3555.

[7] J. D. Bekenstein, Lett. Nuovo Cimento, 4:2 (1972), 737-740; Phys. Rev. D, 7:8 (1973), 2333-2346; 9:12 (1974), 3292-3300.

[8] J. M. Bardeen, B. Carter, S. W. Hawking, Commun. Math. Phys., 31:1 (1973), 161-170.

[9] S. W. Hawking, Nature, 248:5443 (1974), 30-31; Commun. Math. Phys., 43:3 (1975), 199-220.

[10] W. G. Unruh, Phys. Rev. D, 14 (1976), 870-892.

[11] J. D. Bekenstein, Lett. Nuovo Cimento, 11:2 (1974), 467-470.

[12] J.D. Bekenstein, V.F. Mukhanov, Phys. Lett. B, 360:1-2 (1995), 7-12, arXiv: gr-qc/9505012.

[13] A. Ashtekar, J. Baez, A. Corichi, K. Krasnov, Phys. Rev. Lett., 80:5 (1998), 904-907, arXiv: gr-qc/9710007.

[14] A. Ashtekar, J. Baez, K. Krasnov, Adv. Theor. Math. Phys., 4:1 (2000), 1-94. 
[15] S. Hod, Phys. Rev. Lett., 81:20 (1998), 4293-4296, arXiv: gr-qc/9812002.

[16] L. Motl, Adv. Theor. Math. Phys., 6:6 (2003), 1135-1162, arXiv: gr-qc/0212096.

[17] L. Motl, A. Neitzke, Adv. Theor. Math. Phys., 7:2 (2003), 307-330.

[18] J. Natario, R. Schiappa, Adv. Theor. Math. Phys., 8:6 (2004), 1001-1131, arXiv: hep-th/0411267.

[19] V.A. Berezin, Phys. Lett. B, 241:2 (1990), 194-200.

[20] V.A. Berezin, A. M. Boyarsky, A. Yu. Neronov, Phys. Rev. D, 57:2 (1998), 1118-1128, arXiv: gr-qc/9708060.

[21] V. A. Berezin, M. Yu. Okhrimenko, Class. Quant. Grav., 18:11 (2001), 2195-2215, arXiv: gr-qc/0006085. 